\section{The basis of the Bourdon illusion}

\section{PETER WENDEROTH \\ University of Sydney, Sydney, New South Wales, Australia}

Day (1990) and Day, Mitchell, and Stecher (1990) recently reported new data on the Bourdon illusion, showing that the effect occurs in novel variations of the classic figure-for example, with orthogonal and parallel test edges-and also that it occurs in the haptic modality. Day (1990) criticized theories of the Bourdon effect proposed by Wenderoth, Criss, and van der Zwan (1990), Wenderoth and O'Connor (1987a, 1987b), and Wenderoth, O'Connor, and Johnson (1986), and proposed his own "perceptual compromise" hypothesis. It is argued that Day has inaccurately portrayed the Wenderoth et al theory, and that his own hypothesis lacks predictive power because it lacks any reference to neural mechanisms. Day's theory is thus unable to account for extant data, including his own. It is demonstrated how Day's novel experiments provide insights into the mechanisms of the Bourdon effect, and also how the Wenderoth et al. theory can account for the new data of Day (1990) and Day et al. (1990).

Day (1990) and Day, Mitchell, and Stecher (1990) recently presented new data on the Bourdon illusion, and Day (1990) criticized others' theories of the effect and expounded his own theory. This paper aims, first, to correct some inaccuracies in Day's (1990) interpretation of our theory (Wenderoth, O'Connor, \& Johnson, 1986; Wenderoth \& O'Connor, 1987a, 1987b; Wenderoth, Criss, \& van der Zwan, 1990); second, to show that Day's theory lacks predictive power; third, to show how our theory can easily explain differences between conditions used by Day et al. (1990) which cannot be explained by Day's own theory; and, fourth, to use the data from Day's novel experiments to explain previously puzzling data on the Bourdon effect, such as why it peaks at $22.5^{\circ}$ from vertical or horizontal.

If two identical solid scalene triangles are placed apex to apex so that the longest sides are aligned (as in Figure 1A), the straight edge so formed appears bent in the direction of the opposite sides. Three sets of theories recently have been formulated to account for this illusion, which is known as the Bourdon effect.

Day (1990) attempted to explain the effect in terms of a general concept which he has applied elsewhere, a process to which he refers as perceptual compromise. Walker and Shank $(1987,1988 \mathrm{a}, 1988 \mathrm{~b})$ proposed two theories: one involved interactions between orientation

This research was supported by the Australian Research Council, Grant A78831871. Thanks are extended to Ross Day, Christopher Tyler, and an unknown referee for their comments on the manuscript. The author's mailing address is Department of Psychology, University of Sydney, Sydney, N.S.W. 2006, Australia. selective neurons, and the other suggested that the test (straight) and inducing (bent) sides of the Bourdon figure are confused with each other. Wenderoth et al. (1986; Wenderoth \& O'Connor, 1987a, 1987b; Wenderoth, Criss, \& van der Zwan, 1990) suggested that the illusion is a manifestation of conflicting signals arising in separate and parallel neural channels coding orientation, position, and collinearity. However, Wenderoth and his colleagues have also used the term confusion.

Day (1990) criticized both Walker and Shank (1987) and Wenderoth and O'Connor (1987b) for the use of this term stating that "the orientation of the test edge ... the figure (or object) and the opposite edge ... are entirely discriminable. There is no sense in which they are mistaken for, or confused with, each other ... an explanation ... in terms of confusion between different orientations is no more than an alternative description of it" (p. 404).

One can but agree completely with Day that it would be foolish to assert that the left and right edges and the figural content in Figure 1A are not discriminable. However, while I cannot speak on behalf of Walker and Shank, we certainly have never made such an assertion. Certainly, we have used the term "confusion" but always have noted that this is a shorthand way of referring to a percept arising from the output of separate and parallel neural channels coding orientation, position, and collinearity. For example, when Wenderoth and O'Connor (1987a) explicitly use the word "confusion," they clearly state that this is a shorthand reference to the Wenderoth and O'Connor (1987b) compromise hypothesis. Thus:

The solid illusion ... involves a failure to discriminate adequately the orientation of the judged edge from the orientations of the bisectors of the angles ... this is ... a shorthand way of describing a confusion between the judged edges and other parts of the figure ... experiments conducted so far were designed to establish whether the explanation of solid-angle assimilation illusions is to be sought amongst the general category of confusion effects, one version of which is the Wenderoth and O'Connor (1987) compromise hypothesis." (Wenderoth \& O'Connor, 1987a, p. 81; emphasis mine)

This "Wenderoth and O'Connor (1987) compromise hypothesis" restates the view of Wenderoth, O'Connor, and Johnstone (1986) that whereas parallel and separate neural channels coding orientation, position, and collinearity normally carry neural signals that do not conflict, this is not the case with the Bourdon test edge, and so a neural, and therefore perceptual, compromise occurs. Wenderoth and O'Connor (1987b, p. 45) state:

On the basis of other evidence from studies of the tilt illusion and aftereffect ... Wenderoth, O'Connor, and Johnson (1986c) interpreted their data as consistent with the hypothesis that perceptual judgments represent a compromise between separate neural channels that code orientation, po- 
sition, and collinearity. Thus, in chevron matching to outline displays, the individual angle expansion effects are counteracted by information about the straightness of the whole edge ... and by the positional attraction ... toward ... nearby contours.... In solid displays, both the orientation and positional effects are negative, and perceptually it is difficult to isolate the edge ... from the rest of the figure. Hence, all of these effects reinforce each other in the chevron task, resulting in a large negative illusion.

In retrospect, while it is true that our use of terms like "confusion" as a shorthand term for this theory was unfortunate, nevertheless it is clear that the theory involves much more than a simple failure to discriminate one edge
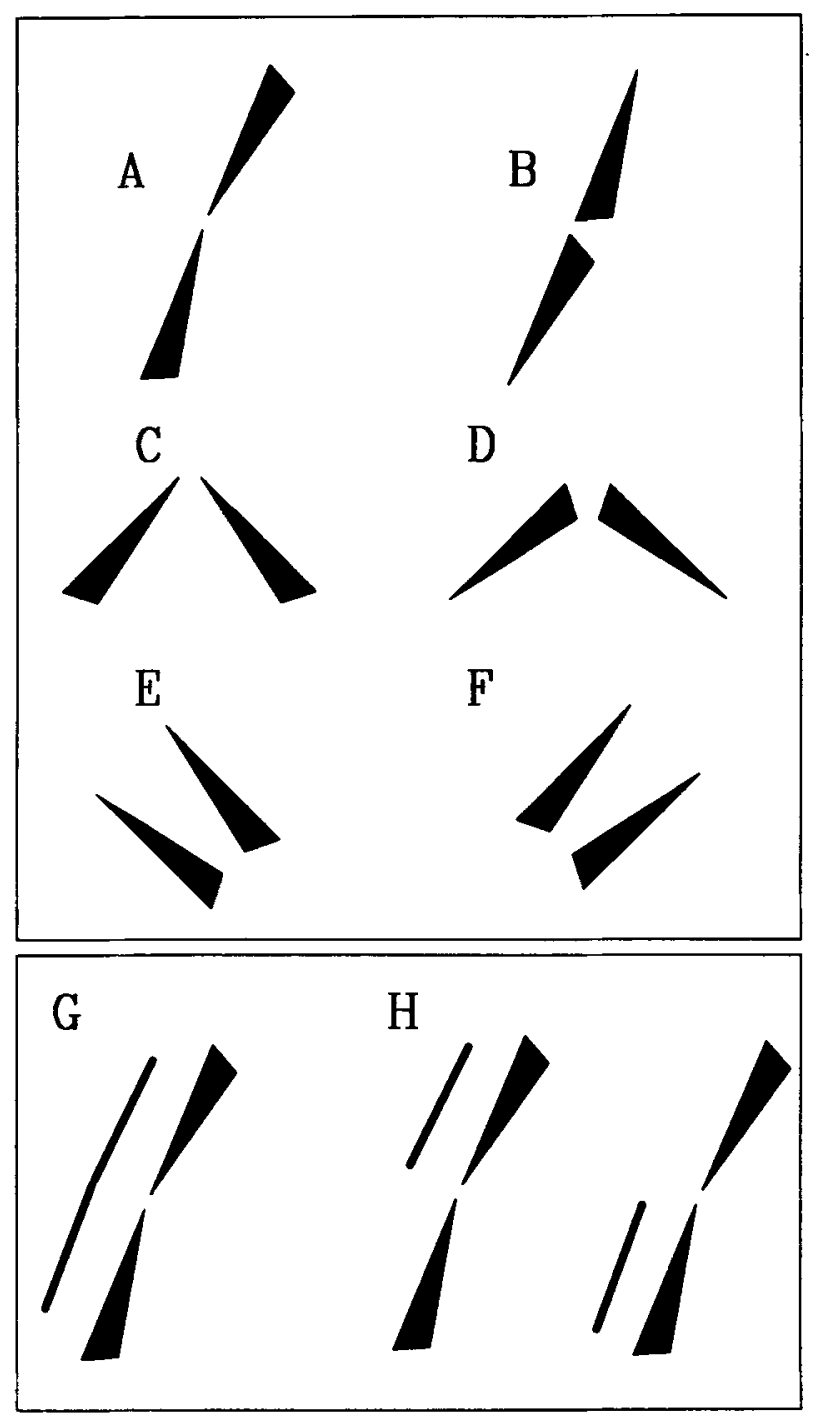

Figure 1. (A) The Bourdon illusion: The left edge is straight but appears bent. (A-F) Bourdon variants used by Day, Mitchell, and Stecher (1990). (G) Chevron matching technique used by Wenderoth et al. $(1986,1987 \mathrm{a}, 1987 \mathrm{~b}, 1990)$ to measure the Bourdon effect. (H) Single upper and lower parallel matching method used by Wenderoth et al. to measure the orientation illusion component of the Bourdon illusion. from another. In the Müller-Lyer figure, for example, it is difficult perceptually to isolate the shafts from the arrowheads; yet in no sense does the observer confuse the shafts and arrowheads.

Without restating the logic or the outcomes of all of the 13 experiments we have reported in four papers to date, it is important to recognize that this neural compromise theory has successfully predicted and/or explained several empirical results, most new. The major variable, the difference between solid and outline figures, is explained by postulating that collinearity cues are more salient in the outline figure, thus reducing the effect; and that position effects are directionally opposite in outline figures but are in the same direction in solid figures (Wenderoth \& O'Connor, 1987b, p. 45). Furthermore, the directionally opposite orientation effects have been demonstrated using the orientation matching task (Wenderoth et al., 1986). Thus, directionally opposite effects to those in Figure 1A occur with outline rather than solid figures and are much smaller in magnitude; similar effects occur if these figures consist of single wedges rather than double wedges (as in Figure 1A); solid double-wedge effects more than exceed the sum of the individual wedge effects, but outline double-wedge effects are less than the sum of the component effects; large and significant solid-angle Bourdon effects occur only when the judged edge is physically straight, not vertical or horizontal, and relatively small in visual angle terms, none of which is true of outline effects; and manipulations which reduce acuity for parallelism increase solid-angle effects but reduce or even reverse the direction of outline-angle effects.

Before considering Day's alternate theory, it needs to be noted that he also states that "Wenderoth and O'Connor (1987) concluded that the Bourdon illusion does not derive directly from neural interactions" (Day, 1990, p. 404). Wenderoth and O'Connor (1987b) actually said that "these six experiments strongly suggest that solid-angle illusions ... are not neurally based effects in the way that outline tilt illusions are thought to be"' (p. 51, emphasis mine). They went on to say that this conclusion derived from the fact that the independent variables in their experiments differentially affected outline and solid-angle effects. That is, they were asserting that the neural mechanisms of solidangle effects were not the same neural mechanisms that underlie the tilt illusion. In fact, I have argued consistently that perceptual processes can only possibly reflect neural interactions directly, and that attempts to distinguish between neurally based effects and "higher order" or "hypothesis testing" processes are meaningless and obscurantist, and reflect current ignorance of mechanisms rather than their nonexistence (Wenderoth \& Johnstone, 1987; Wenderoth \& Latimer, 1978). It can be noted in addition that the idea of separate neural channels coding orientation, position, and collinearity was adapted from the same model put forward to explain tilt aftereffect data in an entirely different context (Harris \& Calvert, 1985).

Turning now to Day's (1990) theory, he states his theory of the haptic (and the visual) Bourdon effect as follows: 
As proposed for the visual Bourdon effect ... the essence of the explanation is that frequently there are numerous cues for a variety of stimulus properties, such as edges, depth, and form. When the cues for a particular property are deliberately contrived to be in conflict, some signaling one state of affairs and others a different one, the outcome in perception will be a compromise between them.... [In the Bourdon illusion] the edges are oriented in one direction, and the components as represented by their bisectors in another. In consequence, there is a compromise between the two orientations, so that the test edges appear to be oriented slightly in the direction of the components. " (Day, 1990 , p. 404)

Consider the question of predictive/explanatory power. The reason that the Wenderoth et al. theory was able to predict the results outlined above was that it drew upon known properties of neural mechanisms coding stimulus features. For example, it explained differences between outline- and solid-angle effects partly in terms of tilt illusions arising from inhibitory interactions between orientation-selective channels in outline displays but their absence in solid displays; and it explained the occurrence of outline effects but not solid effects at the cardinal axes in the same way, with the additional effects of more broadly tuned channels at oblique orientations. I would claim that Day's theory is essentially identical to ours, with the important exception that it lacks entirely any reference to neural mechanisms. It therefore is unable to predict differences between outline- and solid-angle effects, the reduction of Bourdon effects when the test edge is bent, the absence of solid but not of outline effects at vertical and horizontal, the nonlinear summation of singleto double-wedge effects, and so on. What is required is that Day's theory be extended to incorporate propositions that allow the deduction of the magnitude of perceptual compromise under a variety of stimulus conditions.

Consider an example from Day et al.'s (1990) recent data. They measured the Bourdon illusion using a variety of ingenious variations on the original figure: these are shown in Figure 1. Day et al. rightly asserted that the effect should be equally evident, but directionally opposite, when the wedge bases, rather than their apices, are juxtaposed (Figure 1B), and that the effect should also occur when subjects judge either truly orthogonal edges (Figures $1 \mathrm{C}$ and $1 \mathrm{D}$ ) or truly parallel edges (Figures $1 \mathrm{E}$ and $1 F$ ).

These results were obtained. However, for both the visual (Day et al., 1990) and the haptic effects (Day, 1990), the Figure 1B display resulted in a significantly smaller effect than the Figure 1A display. Also, the visual effect for Figure 1D was about half that for Figure 1C, and both right-angle effects exceeded their collinear analogs. Finally, the effect in Figure $1 F$ exceeded that in Figure 1E. Day et al. (1990) correctly point out that this last result probably relates to the apparent divergence of the upper ends of oblique parallels, which, for unknown reasons, is greater for right-tilted lines (Carpenter \& Blakemore, 1973; Rochlin, 1955).
But what accounts for the differences between the other displays? I agree with Day et al.'s claim that the average effects in right-angle figures exceeded those in aligned figures because the cue of collinearity had been removed. But Day's theory cannot explain the smaller effects in Figures $1 B$ and $1 D$ as compared with those of Figures $1 \mathrm{~A}$ and 1C. Day et al. discuss the possibility of "affordances of bending," dismiss it, and conclude that the reason for the differences is at present unclear.

We have noted (Wenderoth, Criss, \& van der Zwan, 1990; Wenderoth \& O'Connor, 1987a, 1987b; Wenderoth et al., 1986) that when subjects were required to set a chevron placed adjacent to the Bourdon edge to match it (Figure 1G), most observers (including myself) tended first to try to set the upper chevron arm parallel to the edge of the upper wedge, to repeat this for the lower edge, and then to make a second-stage, global judgment in which the matching chevron was bent even more. This two-stage process manifested itself in the finding that chevron matches were greater than the sum of individual and single parallel matches (Figure $1 \mathrm{H}$ ) to each wedge individually (Wenderoth et al., 1986).

In terms of our theory, the first stage of the two-stage adjustment involves orientation judgment, and the second involves relative position and collinearity of the two wedges. It is noteworthy that our single-wedge parallel matching errors averaged about $1^{\circ}$ and the effect Day obtained for the Figure 1B display, both visually and haptically, was about $1.5^{\circ}$. I would suggest that the effects in the different displays shown in Figures 1A to $1 D$ are ordered as they are for the following reasons. Figure 1C gives the largest illusion because cues to collinearity are lacking, the apex-to-apex arrangement adds a position effect (explained below), and the solid wedges add the usual orientation effect. The next largest illusion occurs in Figure 1D, because it does not contain collinearity cues and it does contain the orientation effect, but the position effect has been removed by rearranging the figure to juxtapose the bases. In Figure 1A, where the next largest effect occurs, collinearity cues now reduce the effect of orientation and position effects, which are both present, the position effect because the apices are adjacent. Finally, the rearrangement of the bases in Figure $1 \mathrm{~B}$ removes the position effect, leaving only the orientation component reduced by collinearity cues.

What, then, is the position effect? The position effect can be seen in Figures 1A and 1B: the upper and lower extremities of the wedges appear displaced to the right relative to the center in Figure 1A, but these same thickwedge extremities do not appear to be displaced to the right when they are centrally adjacent in Figure 1B: their adjacency provides cues for position. Thus, the position effect is independent of the orientation effect that occurs in all single- as well as double-wedge figures. This is consistent with other data, both mine and Day's, which show the independence of judgments in different domains such as orientation, position, angle, and length (Day, Bellamy, \& Norman, 1983; Wenderoth, 1983; Wenderoth, Parkin- 
son, \& White, 1979; Wenderoth \& White, 1979). If this analysis is correct, it can be predicted further that subjects required to make chevron matches to Figure 1B stimuli would not make the second-stage adjustment which, in that task, reflects the position effect: first-stage orientation matches would also be acceptable to the subject as a double-wedge match, and the double-wedge chevron match would then be the sum of upper and lower single-wedge matches.

Finally, Wenderoth and O'Connor (1987a) showed that solid-angle Bourdon effects increase with separation between the wedges, which they explained by the fact that separation decreases collinearity cues. They predicted, and found, that outline-angle effects reversed direction with separation, as position effects, no longer overridden by collinearity cues, began to more than offset directionally opposite tilt illusions. Day et al. (1990) presented similar informal demonstrations, but additionally noted that the adjacent base figure (Figure 1B) produced a large apparent bending effect when the gap between the bases was large. The account given here explains that result, too, because separating the bases reduces cues to the relative positions of the bases.

\section{SUMMARY AND CONCLUSIONS}

It has been argued that Day's (1990) representation of Wenderoth et al.'s (Wenderoth et al., 1986; Wenderoth \& O'Connor, 1987a, 1987b; Wenderoth et al., 1990) theory of the Bourdon illusion is inaccurate, in that it attributes claims to those authors which they have not made, and it fails to take into account the neural basis of the theory, couched in terms of separate and parallel neural channels coding orientation, position, and collinearity. It has been demonstrated how this theory can explain data presented by Day (1990) and Day et al. (1990) for which his own theory cannot account, because it lacks a mechanistic basis and, hence, explanatory and predictive power. The fact that the Bourdon effect occurs in the haptic modality may suggest that separate and parallel channels exist in that modality, similar to those which we have postulated to account for the visual effect, although the possibility cannot be ruled out that subjects visualize during haptic judgments and that this in turn somehow triggers visual mechanisms (Wenderoth \& Alais, 1990).

The analysis presented here also has strong implications for methods of measuring the Bourdon effect and its variants. For example, Day et al. (1990) presented a figure in which the right-hand edges of a Bourdon display, such as that in Figure 1A, were curved rather than tilted, and reported anecdotally that a slight apparent curvature was evident in the test edges but that they appeared aligned. Day et al. speculated that this might reflect a lack of interaction between orientation and curvature-selective mechanisms. In terms of our theory, it can be suggested that the curved wedges induce a curvature illusion but not a position effect. This could be tested by using an analogue of the chevron matching technique with single- and double-wedge matching to determine whether there is a second-stage adjustment. I suggest that it is highly likely that, unless very careful instructions are given to subjects, measurement techniques that require observers to select a matching angle (e.g., Walker \& Shank, 1987, 1988a, $1988 \mathrm{~b}$ ) or to adjust the Bourdon wedges themselves (e.g., Day et al., 1990) may confound orientation and position effects. This might explain the fact that "unbending" effects (e.g., Walker \& Shank, 1987, 1988a, 1988b) measured on the right-hand edges of Figure $1 \mathrm{~A}$ are very small when chevron matching is used, but much larger when a match is selected from a series of angles, as we have found (Wenderoth et al., 1990).

Finally, it has always been somewhat puzzling that the Bourdon effect is largest when the figure is oriented $22.5^{\circ}$ from vertical or horizontal (Rozvany \& Day, 1980; Wenderoth \& O'Connor, $1987 \mathrm{~b}$ ), because orientation illusions peak at $45^{\circ}$ (Carpenter \& Blakemore, 1973; Wenderoth \& O'Connor, 1987b). When Bouma and Andriessen (1968) had subjects align a distant dot with the perceived extension of an oriented line segment, their obtained alignment errors peaked at $22.5^{\circ}$. Although they believed they were measuring perceived line orientation, subsequent research suggested that their task did not measure perceived orientation (Wenderoth et al., 1979) and that dot alignment "is more like a position-domain than an orientation-domain judgment" (Wenderoth, 1983, p. 401). If position illusions peak at $22.5^{\circ}$, and since tilt illusions at $22.5^{\circ}$ are not markedly different from those at $22.5^{\circ}$ or $67.5^{\circ}$, the Bourdon effect might peak at $22.5^{\circ}$ from the cardinal axes because this maximizes the sum (not necessarily linear) of orientation and position illusions.

\section{REFERENCES}

Bouma, H., \& ANDRIESSEN, H. H. (1968). Perceived orientation of isolated line segments. Vision Research, 8, 493-507.

CARPENTER, R. H. S., \& Blakemore, C. (1973). Interactions between orientations in human vision. Experimental Brain Research, 18, 287-303.

Day, R. H. (1990). The Bourdon illusion in haptic space. Perception \& Psychophysics, 47, 400-404.

Day, R. H., Bellamy, S., \& Norman, A. (1983). On the Morinaga illusion. Journal of Experimental Psychology: Human Perception \& Performance, 9, 113-125.

Day, R. H., Mitchell, P., Stecher, E. J. (1990). The Bourdon illusion occurs with straight-, right-angle, and parallel-edge figures. Perception \& Psychophysics, 48, 375-381.

HarRis, J. P., \& CalverT, J. E. (1985). The tilt after-effect: Changes with stimulus size and eccentricity. Spatial Vision, 1, 113-129.

Rochlin, A. M. (1955). The effect of tilt on the visual perception of paralleiness. American Joumal of Psychology, 68, 223-236.

Rozvany, G. E., \& Day, R. H. (1980). Determinants of the Bourdon effect. Perception \& Psychophysics, 28, 39-44.

Walker, J. T., \& Shank, M. D. (1987). The Bourdon illusion in subjective contours. Perception \& Psychophysics, 42, 15-25.

Walker, J. T., \& ShANK, M. D. (1988a). Interactions between real and subjective contours in the Bourdon illusion. Perception \& Psychophysics, 43, 567-574.

Walker, J. T., \& Shank, M. D. (1988b). Real and subjective lines and edges in the Bourdon illusion. Perception \& Psychophysics, 43. 475-484.

WENDEROTH, P. (1983). Identical stimuli are judged differently in the 
orientation and position domains. Perception \& Psychophysics, 33, 399-402.

Wenderoth, P., \& Alais, D. (1990). Lack of evidence for a tactual Poggendorff illusion. Perception \& Psychophysics, 48, 234-242.

Wenderoth, P., Cruss, G., \& VAN der Zwan, R. (1990). Determinants of subjective contour Bourdon illusions and "unbending" effects. Perception \& Psychophysics, 48, 497-508.

Wenderoth, P., \& Johnstone, S. (1987). Possible neural substrates for orientation analysis and perception. Perception, 16, 693-709.

WENDEROTh, P., \& LATIMER, C. (1978). On the relationship between the psychology of visual perception and the neurophysiology of vision. In J. P. Sutcliffe (Ed.), Conceptual analysis and method in psychology: Essays in honour of W. M. ONeil (pp. 106-117). Sydney: Sydney University Press.

Wenderoth, P., d O'Connor, T. (1987a). The effects of display and observer strategy variables on Bourdon assimilation illusions. Perception, 16, 79-88.
Wenderoth, P., O'Connor, T. (1987b). Outline- and solid-angle illusions have different determinants. Perception \& Psychophysics. 41, 45-52.

Wenderoth, P., O'Connor, T., Johnson, M. (1986). Expansion and contraction of outline and solid acute angles: effects of angle magnitude, type of display, and the nature of the matching task. Perception \& Psychophysics, 39, 261-266.

Wenderoth, P., Parkinson, A., \& WhITE, D. (1979). A comparison of visual tilt illusions measured by the techniques of vertical setting. parallel matching and dot alignment. Perception, 8, 47-57.

Wenderoth, P., White, D. (1979). Angle matching illusions and perceived line orientation. Perception, 8, 565-575.

(Manuscript received June 14, 1990; revision accepted for publication November 19, 1990.)

\section{Perception \& Psychophysics Articles In Press}

The following is a list of forthcoming Perception \& Psychophysics articles that are currently in press. They are given in approximate order of acceptance. Each entry includes the name and address of the author with whom to communicate for further prepublication information.

"The Fraser illusion: Complex figures" by G. W. Stuart \& R. H. Day (G.W.S., Ctr. for Vis. Sci., Australian Nat'l. Univ., GPO Box 475, Canberra, ACT 2061, Australia)

"Observations on the adaptation of induced motion" by $\mathbf{R}$. B. Post \& L. A. Lott (either author, Dept. Psychol., Univ. California, Davis, CA 95616)

"Further evidence for a time-independent shift of the focus of attention"' by H.-W. Kwak, D. Dagenbach, \& H. Egeth (H.E., Dept. Psychol., Ames Hall, Johns Hopkins Univ., Baltimore, MD 21218)

"On the locus of redundancy effects in a letter-detection task" by J. G. Rueckl, S. Suzuki, \& S.-L. Yeh (J.G.R., Dept. Psychol., Harvard Univ., Cambridge, MA 02138)

"Time estimation and attentional perspective" by M. Boltz (Dept. Psychol., Haverford Coll., Haverford, PA 19041)

"Normal McCollough effect in Alzheimer's disease and global amnesia"' by R. L. Savoy \& J. D. E. Gabrieli (R.L.S., Rowland Inst. for Sci., 100 Cambridge Pkwy., Cambridge, MA 02142)

"Importance of head axes in perception of cutaneous patterns drawn on vertical body surfaces"' by K. Sekiyama (Dept. Psychol., Kanazawa Univ., Kakuma-machi, Kanazawa, 920-11, Japan)

"Contingent-aftereffects: Lateral interactions between color and motion" by L. T. Sharpe, J. P. Harris, C. C. Fach, \& D. I. Braun (L.T.S., Neurologische Universitätsklinik, Hansastr. 9, D-7800 Freiburg im Breisgau, Germany)

"Time course of recovery from gustatory adaptation to $\mathrm{NaCl}$ " by Z. Bujas, S. Szabo, D. Ajduković, \& D. Mayer (S.S., Dept. Psychol., Univ. Zagreb, Zagreb, Yugoslavia)

"Apparent reversals of a rotating mask: A new demonstration of cognition in perception" by D. S. Klopfer (Dept. Psychol., Bowling Green St. Univ., Bowling Green, OH 43403)

"Manual discrimination of force using active finger motion" by X. D. Pang, H. Z. Tan, \& N. I. Durlach (Rsch. Lab. of Electronics and Dept. Electrical Eng. \& Computer Sci., MIT, Cambridge, MA 02139)

"The effect of training on visual alignment discrimination and grating resolution" by R. G. Bennett \& G. Westheimer (R.G.B.,
Dept. Psychol., Murdoch Univ., Murdoch, WA 6150, Australia)

"Perceptual order and the effect of vocalic context on fricative perception" by V. Mann \& S. D. Soli (V.M., Dept. Cog. Sci., Univ. California, Irvine, CA 92717)

"Motion capture by a frame-global or local processing?" by B. Gilliam \& R. Broughton (B.G., Sch. Psychol., Univ. New South Wales, P.O. Box 1, Kensington, NSW 2033, Australia)

"A weighted-average model of achromatic transparency" by S. C. Masin (Dept. Gen. Psychol., Univ. Padova, Piazza Capitaniato 3, 35100 Padova, Italy)

"Two-stage model for order discrimination" by P. Jaskowski (Dept. Biophysics, Med. Acad., Fredry 10, 61-701 Poznan, Poland)

"Contrast thresholds for identification of numeric characters in direct and eccentric view" by H. Strasburger, L. O. Harvey, Jr., \& I. Rentschler (H.S., Inst. of Med. Psychol., Goethestr. 31, D-800 Muenchen 2, Germany)

"Does intermittence in induced rotary movement have any explanatory significance?' 'by A. H. Reinhardt-Rutland (Dept. Psychol., Rm. 17J05, Univ. Ulster at Jordanstown, Shore Rd., Newtownabbey, Co. Antrim BT37 0QB, Northern Ireland)

"The influence of parafoveal preprocessing and linguistic context on the optimal landing position effect" by F. Vitu, Ctr. for the Study of Reading, 51 Gerty Dr., Champaign, IL 61820)

"Visual selection of features and objects: Is location special? A reinterpretation of Nissen's (1985) findings' by C. Bundesen (Psychol. Lab., Copenhagen Univ., Njalsgade 90, DK-2300 Copenhagen S., Denmark)

"Sensorimotor synchronization: Motor responses to regular auditory patterns' 'by M. Franek, J. Mates, T. Radil, K. Beck, \& E. Pöppel (M.F., Inst. Musicol., Na Perśtýne 1, Praha 1, CS 11001 , Czechoslovakia)

"An 'open-transformed scale' for correcting ceiling effects and enhancing retest reliability: The example of pain" by $\mathbf{E}$. Fernandez, T. E. Nygren, \& B. E. Thorn (E.F., Dept. Psychol., Univ. Queensland, St. Lucia, QLD 4072, Australia) 